Tropical Journal of Pharmaceutical Research May 2015; 14(5): 879-889

ISSN: $1596-5996$ (print); 1596-9827 (electronic)

(c) Pharmacotherapy Group, Faculty of Pharmacy, University of Benin, Benin City, 300001 Nigeria.

All rights reserved.

Available online at http://www.tjpr.org

Original Research Article

http://dx.doi.org/10.4314/tjpr.v14i5.19

\title{
Simultaneous Determination of Flavonols and Terpene Lactones in Beagle Dog Plasma by Ultra-Performance Liquid Chromatography-Tandem - Mass Spectrometry: 2. Application to Pharmacokinetic Studies on Ginkgo Leaf Extract
}

\author{
Yang Lu, Pengyue Li, Huimin Liu, Shouying Du*, Yanke Cheng, Huichao Wu, \\ Jiannan Feng and Beibei Shao \\ School of Chinese Materia Medica, Beijing University of Chinese Medicine, Beijing 100102, China
}

*For correspondence: Email: dushouying@263.net; Tel: 010-84738615

Received: 25 August 2014

Revised accepted: 6 April 2015

\begin{abstract}
Purpose: To evaluate the pharmacokinetics of the major compounds in Ginkgo leaf dosage formulations (namely, Yikangning tablets, Ginaton tablets, Aoshi dropping pills and Yinxinke dispersible tablets), commonly used in traditional Chinese medicine.

Methods: $A$ randomized $4{ }^{*} 4$ crossover study with eight beagle dogs was carried out. Plasma samples were collected following oral administration of four different preparations and the effective ingredients, namely, kaempferol, quercetin, isorhamnetin, ginkgolides $A$, ginkgolides $B$, ginkgolides $C$ and bilobalide were detected by a validated ultra-performance liquid chromatography-tandem mass spectrometry (UPLC-TMS). Then the pharmacokinetics of these target compounds, of different preparations were studied.

Results: The adjusted pharmacokinetic data showed that the area under the concentration-time curve from time-zero to the last measurable concentration $\left(A \cup C_{0-t}\right)$ of kaempferol, quercetin, isorhamnetin, bilobalide, ginkgolides $A$, ginkgolides $B$, and ginkgolides $C$ in plasma ranged from $124.37 \pm 90.46$ to $2261.87 \pm 812.35$, after administration of Yikangning; $142.28 \pm 62.37$ to $2529.46 \pm 320.48 \mu \mathrm{g} / \mathrm{L} \cdot \mathrm{h}$ following administration of Ginaton; $158.52 \pm 55.48$ to $1987.40 \pm 766.21 \mu \mathrm{g} / \mathrm{L} \cdot \mathrm{h}$ after Aosh administration; $160.49 \pm 104.66$ to $2016.92 \pm 1150.92 \mu \mathrm{g} / \mathrm{L} \cdot \mathrm{h}$ following Yinxinke administration. The results also indicate that the flavonoids (especially quercetin) in dispersible tablets and dropping pills hexhibited higher AUC than those in conventional tablets. There were no differences between Aosh (dropping pills) and Yinxinke (dispersible tablets) in terms of the bioavailability of the flavonoids, but the dropping pill flavonoids showed lower $t_{\max }$.

Conclusion: The results indicate that UPLC-TMS can used to simultaneously evaluate the plasma pharmacokinetics of Ginko compounds in beagle dogs
\end{abstract}

Keywords: Ginkgo biloba, Beagle dog plasma, Kaempferol, Quercetin, Isorhamnetin, Ginkgolides A, Ginkgolides B, Ginkgolides C, Bilobalide, Pharmacokinetics; Bioavailability

Tropical Journal of Pharmaceutical Research is indexed by Science Citation Index (SciSearch), Scopus, International Pharmaceutical Abstract, Chemical Abstracts, Embase, Index Copernicus, EBSCO, African Index Medicus, JournalSeek, Journal Citation Reports/Science Edition, Directory of Open Access Journals (DOAJ), African Journal Online, Bioline International, Open-J-Gate and Pharmacy Abstracts 


\section{INTRODUCTION}

Ginkgo probably originated thousands of years ago. Ginkgo biloba leaf extracts (GBE) contain 72 ginkgo constituents, including terpene lactones, flavonols, flavones, isoflavones, biflavones, flavanols, and carboxylic acids. The pharmacological activities of GBE are attributed to the synergistic action of terpene lactones and flavonoid glycosides. The products of GBE have become widely used as botanical medicines and dietary supplements, especially for the prevention and treatment of cardiovascular diseases [1] and cerebral insufficiency [2-6].

One of the challenges in clinical application of GBE preparations is the low bioavailability [7] (10 $\%$, poor solubility, poor permeability [8] and the physical problem of delivering a drug across the blood-brain barrier. Hence, many new formulations emerged in the recent years, such as, phospholipid, complexes, tablets [9], solid dispersions [10], and dropping pills. Each formulation has both advantages and disadvantages. For conventional tablets, their technology is sample and auto-operation but they are really hard to take for people who have difficulty swallowing.

Solid dispersible tablets can disintegrate and disperse quickly in the water and in mouth. They have the features of fast acting and high pharmacological effect. However, the solid dispersible tablets must add amount of disintegrants and use micronized raw medicinal material. Dropping pills are prepared by the solid dispersion technology. The process has the advantages of simple equipment, easy control, high efficiency and low cost. However, dropping pills have poor drug loading.

Are new preparations better than conventional tablets in clinical practice? So far, many studies were carried out on preparation methods and in vitro dissolution of new preparations, but few in vivo studies have been done. According to the Food and Drug Administration of the United States, botanical drug products should be investigated with regard to blood levels of known representative markers, active constituents, and/or major chemical constituents. However, so far, the pharmacokinetic studies of GBE have been carried on mostly in rats, and were deficient in comparisons of different preparations [11-14].

The aim of this study was to evaluate the the pharmacokinetic profiles of four different GBE formulations - Yikangning tablets, Ginaton tablets, Aoshi dropping pills, and Yinxinke dispersible tablets - after a single oral administration in beagle dogs, using a previously developed ultrahigh-performance liquid chromatography coupled with triple quadrupole mass (UPLC-MS/MS) to quantify kaempferol (KMF), quercetin (QCT), isorhamnetin (ISR), ginkgolides A (GA), ginkgolides B (GB), ginkgolides $C$ (GC), and bilobalide (BB) simultaneously.

\section{EXPERIMENTAL}

\section{Chemicals and Ginkgo leaf preparations}

The standards of kaempferol (KMF), quercetin (QCT), isorhamnetin (ISR), ginkgolides $A(G A)$, ginkgolides $B(G B)$, ginkgolides $C(G C)$ and bilobalide (BB) and domperidone (DPD) (IS) were purchased from the National Institute for the Control of Pharmaceutical and Biological Products (Beijing, China). The purity of QCT and GC was 96.5 and $97.1 \%$, respectively. The purity of the remaining standards was more than $99 \%$. LC/MS grades of methanol and acetonitrile were obtained from Fisher Scientific (Fair Lawn, NJ, USA), HPLC grade of formic acid was supplied by ROE Scientific (Newcastle, USA), and ultrapure water was generated from the Synergy UV water purification system (Millipore Corp, USA).

Yikangning tablets of $G$. biloba leaves extract were made by Yangtze River Pharmaceutical Group Co. Ltd Jiangsu, China. Each tablet contained $80 \mathrm{mg}$ of the extracts. (Batch: 12062621). Ginaton tablets of $G$. biloba leaves extract were made by Willmar Schwabe $\mathrm{GmbH}$ \& Co. KG (Germany). Each tablet contained $40 \mathrm{mg}$ of the extract. (Batch no. 1941211). Aoshi dropping pills of $G$. biloba leaves extract were made by Zhejiang Jiuxu Pharmaceutical Co., Ltd Zhejiang, China. Each pill contained $10 \mathrm{mg}$ of the extract (Batch no. 20120709). Yinxinke dispersible tablets of $G$. biloba leaves extract were made by Jiangsu Shenlong Pharmaceutical Co., Ltd. (Jiangsu, China). Each tablet contained 40 mg ginkgo extractum (Batch no. 120702).

\section{Instrumentation and chromatographic conditions}

The UPLC-MS/MS system used was composed of an Acquity UPLC system and a TQS triple quadrupole tandem mass spectrometer. Chromatographic separation was performed on an ACQUITY UPLC BEH C18 column. The column temperature was maintained at $40{ }^{\circ} \mathrm{C}$ and the auto-sampler was conditioned at $4{ }^{\circ} \mathrm{C}$. The mobile phase was composed of $0.1 \%$ formic 
acid aqueous solution $(A)$ and acetonitrile $(B)$ at a flow rate of $0.4 \mathrm{~mL} / \mathrm{min}$ in only 4.0 minutes. Gradient condition of the mobile phase was as follows: $5 \% \mathrm{~B}$ at $0-1.0 \mathrm{~min}$; $5 \rightarrow 40 \% \mathrm{~B}$ at $1.0-$ $1.5 \mathrm{~min} ; 40 \% \rightarrow 43 \% \mathrm{~B}$ at $1.5-3.0 \mathrm{~min}$, then the system was equilibrated using the initial condition (acetonitrile-water, 5:95, v/v) for $1.0 \mathrm{~min}$.

\section{Assay of the preparations}

The four ginkgo leaf preparations (GLP) were analyzed by UPLC-MS/MS. The method can be applied to simultaneous determination of KMF, QCT, ISR, BB, GA, GB and GC. Table 1 shows the levels of KMF, QCT, ISR, BB, GA, GB and $\mathrm{GC}$ in the four GLP. Acid hydrolysis was applied to convert flavonoid glycosides into their aglycone forms before measuring the contents of KMF, QCT, and ISR in these preparations.

\section{Pharmacokinetic studies}

This study complied with the Guiding Principles for the Care and Use of Laboratory Animal and was approved by the Institutional Animal Care and Use Committee of the Beijing University of Chinese Medicine (SPF animal, certificate no. SCXK (Jing) 2013-0007).

Eight male beagle dogs weighing $11.1 \pm 0.3 \mathrm{~kg}$ were used. The dogs were housed in a stainless steel cage, with an ambient temperature of 21 $22{ }^{\circ} \mathrm{C}$ and unlimited access to standard laboratory dog diet and water. Except for ad libitum water, the animals were fasted for $12 \mathrm{~h}$ prior to drug administration.

Then a randomized $4 * 4$ crossover trial with eight beagle dogs was carried out to study the pharmacokinetic characteristics. In the design, the equal numbers of subjects are initially assigned to each sequence. Each subject was randomly assigned to any sequence. The advantage of the randomized $4^{*} 4$ crossover study is to reduce the number of animals and improve the data reliability by eliminating the error from the experimental period and animals individuals. The design is presented in Table 2.

Eight male beagle dogs were divided randomly into four groups, and each group was treated with a single dose of Yikangning, Ginaton, Aoshi and Yinxinke (take Ginaton as the reference preparation). Each dog received an oral dose equivalent to $640 \mathrm{mg}$ extract under fasting conditions, that is, 8 tablets of Yikangning, 16 tablets of Ginaton, 64 pills of Aoshi, and 16 tablets of Yinxinke. Drugs were put into the epiglottis of beagle dogs, and then the dogs were made to automatically swallow and drink $50 \mathrm{~mL}$ water. There was a washout period of 2 weeks between the two adjacent periods, and the order of the drug administration was randomized.

Blood samples were collected for up to $48 \mathrm{~h}$ (at $0,10,20,30$ and $45 \mathrm{~min}$, then 1, 1.5, 2, 3, 4, 5, 6, 8, 10, 12, 24, 36, and $48 \mathrm{~h}$ after administration). The blood samples were centrifuged at $5000 \mathrm{rpm}$ for $10 \mathrm{~min}$; then the supernatant plasma were collected into tightly sealed plastic tubes (containing heparin sodium anticoagulant solution), and were finally frozen at - $20^{\circ} \mathrm{C}$ until used for analysis.

All the plasma samples were extracted by using liquid-liquid extraction technique as was described. Hydrochloric acid was added into the samples to convert flavonoid glycosides into their aglycone forms. Accordingly, the measured flavonoid levels were expressed as the concentrations of KMF, QCT and ISR. The samples were prepared as following steps: The plasma sample was hydrolyzed for $30 \mathrm{~min}$ in a

Table 1: Levels of ginkgo compounds in the four GLP

\begin{tabular}{lcccc}
\hline Compound & $\begin{array}{c}\text { Yikangning } \\
\text { mg/tablet }\end{array}$ & $\begin{array}{c}\text { Ginaton } \\
\text { mg/tablet }\end{array}$ & $\begin{array}{c}\text { Aoshi } \\
\text { mg/pill }\end{array}$ & $\begin{array}{c}\text { Yinxinke } \\
\text { mg/tablet }\end{array}$ \\
\hline KMF & 3.95 & 1.36 & 0.51 & 3.49 \\
QCT & 3.39 & 1.36 & 0.45 & 2.79 \\
ISR & 1.59 & 0.38 & 0.24 & 1.25 \\
BB & 3.25 & 1.30 & 0.47 & 2.81 \\
GA & 1.45 & 0.34 & 0.22 & 1.05 \\
GB & 1.03 & 0.30 & 0.19 & 0.70 \\
GC & 1.01 & 0.33 & 0.08 & 0.59 \\
\hline
\end{tabular}

Table 2: A four-period crossover design for drug administration

\begin{tabular}{lcccc}
\hline Period & Sequence A & Sequence B & Sequence C & Sequence D \\
\hline 1 & Yinxinke & Reference & Yikangning & Aoshi \\
2 & Reference & Yinxinke & Aoshi & Yikangning \\
3 & Yikangning & Aoshi & Reference & Yinxinke \\
4 & Aoshi & Yikangning & Yinxinke & Reference \\
\hline
\end{tabular}


water bath at $80^{\circ} \mathrm{C}$ and extracted with acetidin twice, then analyzed by UPLC-MS/MS.

\section{Data analysis}

The pharmacokinetic parameters of different preparations are comparable only if the dogs are treated with the same dosage, but the drug contents (7 effective ingredients) of these preparations were not the same, so a dosage conversion factor was calculated as in Eq 1.

$\mathrm{i}=D_{\mathrm{r}} / \mathrm{D}_{\mathrm{t}}=\left(\mathrm{n}_{\mathrm{r}} \mathrm{m}_{\mathrm{r}}\right) /\left(\mathrm{n}_{\mathrm{t}} \mathrm{m}_{\mathrm{t}}\right)$

where $i$ is the conversion factor, $D_{r}(m g)$ is the administration dosage of reference preparation, $D_{t}(\mathrm{mg})$ is the administration dosage of test preparation, $\mathrm{n}_{\mathrm{r}}$ (tablet or pill) is the number of reference preparation, $\mathrm{m}_{\mathrm{r}}(\mathrm{mg} /$ tablet or $\mathrm{mg} /$ pill) is the content of reference preparation, $n_{t}$ (tablet or pill) is the number of test preparation, and $m_{t}$ $(\mathrm{mg} /$ tablet or $\mathrm{mg} /$ pill) is the content of test preparation.

Second, the conversion factor was multiplied by the measured plasma concentration of QCT, $\mathrm{KMF}$, ISR, BB, GA, GB, and GC to calculate the corresponding plasma concentrations when the dogs are treated with the same dose.

The pharmacokinetic parameters, $\mathrm{AUC}_{0-\mathrm{t}}$ and $T_{1 / 2}$, were calculated with the software program DAS 3.20 (non-compartmental model). Cmax and Tmax were the actual values. Data were analyzed using SPSS 17.0. $A \mathrm{UC}_{0-t}$ and $\mathrm{C}_{\max }$ were compared via analysis of variance (ANOVA) and multiple comparisons (least significance difference, LSD). $\mathrm{T}_{\max }$ was measured using nonparametric statistical tests (Wilcoxon).

The relative bioavailability $\left(F_{r}\right)$ was calculated as in $\mathrm{Eq} \mathrm{2,} \mathrm{using} \mathrm{Ginaton} \mathrm{as} \mathrm{the} \mathrm{reference}$ preparation.

$F_{r}=A U C_{t} / A U C_{r}$

where $A U C_{r}(\mu g / L \cdot h)$ is the area under the concentration-time curve of the reference preparation and $A \cup C_{t}(\mu g / L \cdot h)$ is the area under the concentration-time curve of the test preparation.

\section{RESULTS}

The calculation formulas for the total flavone glycol glycosides and the total lactones according to Chinese Pharmacopoeia (2010 version) and USP 35 - NF 30 are as follows: (1) total flavone glycol glycosides (total flavonoids) = $2.51(\mathrm{KMF}+\mathrm{QCT}+\mathrm{ISR})$ and (2) total lactones $=\mathrm{BB}+\mathrm{GA}+\mathrm{GB}+\mathrm{GC}$. Figs $1-9$ show the mean plasma concentration-time curves of the seven sample total flavonoids, and total terpene lactones in beagle dog plasma after administration of four different GLP. Plasma concentration-time data derived from the experiments were analyzed by DAS 3.20 (noncompartmental model), and the main pharmacokinetic parameters were summarized in Table 3-4.

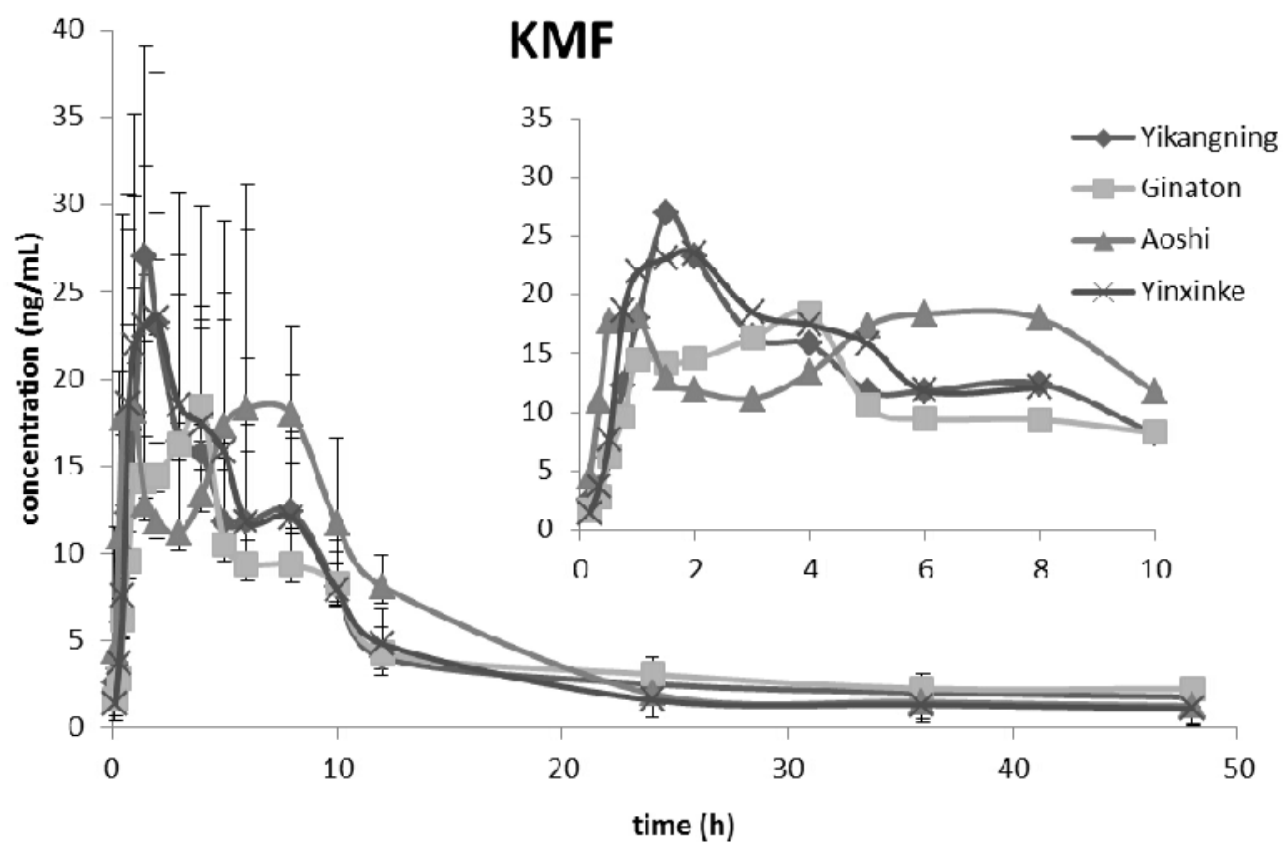

Fig 1: Concentration-time curve of kaempferol (KMF) for different preparations 


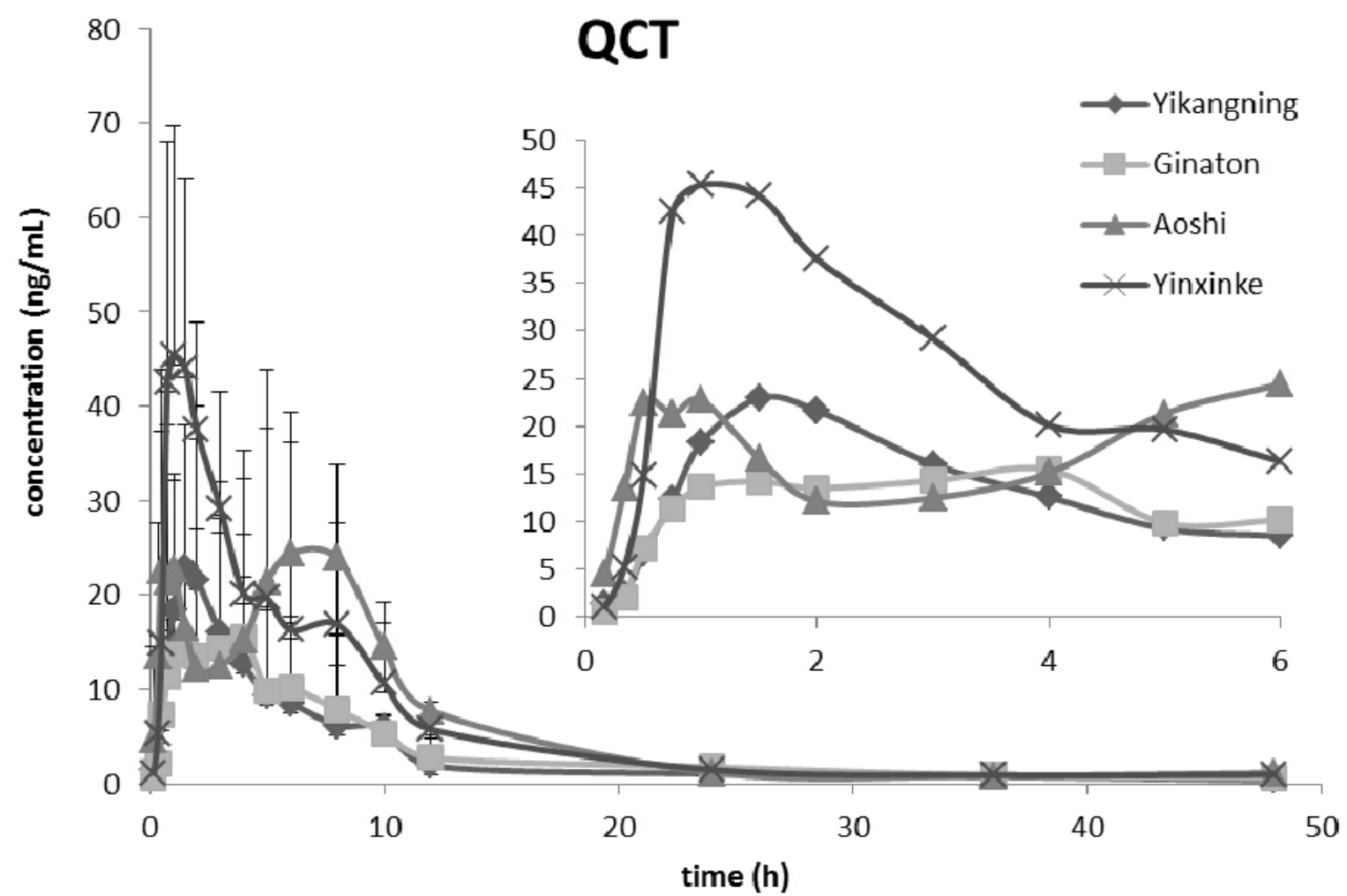

Fig 2: Concentration-time curve of quercetin (QCT) for different preparations

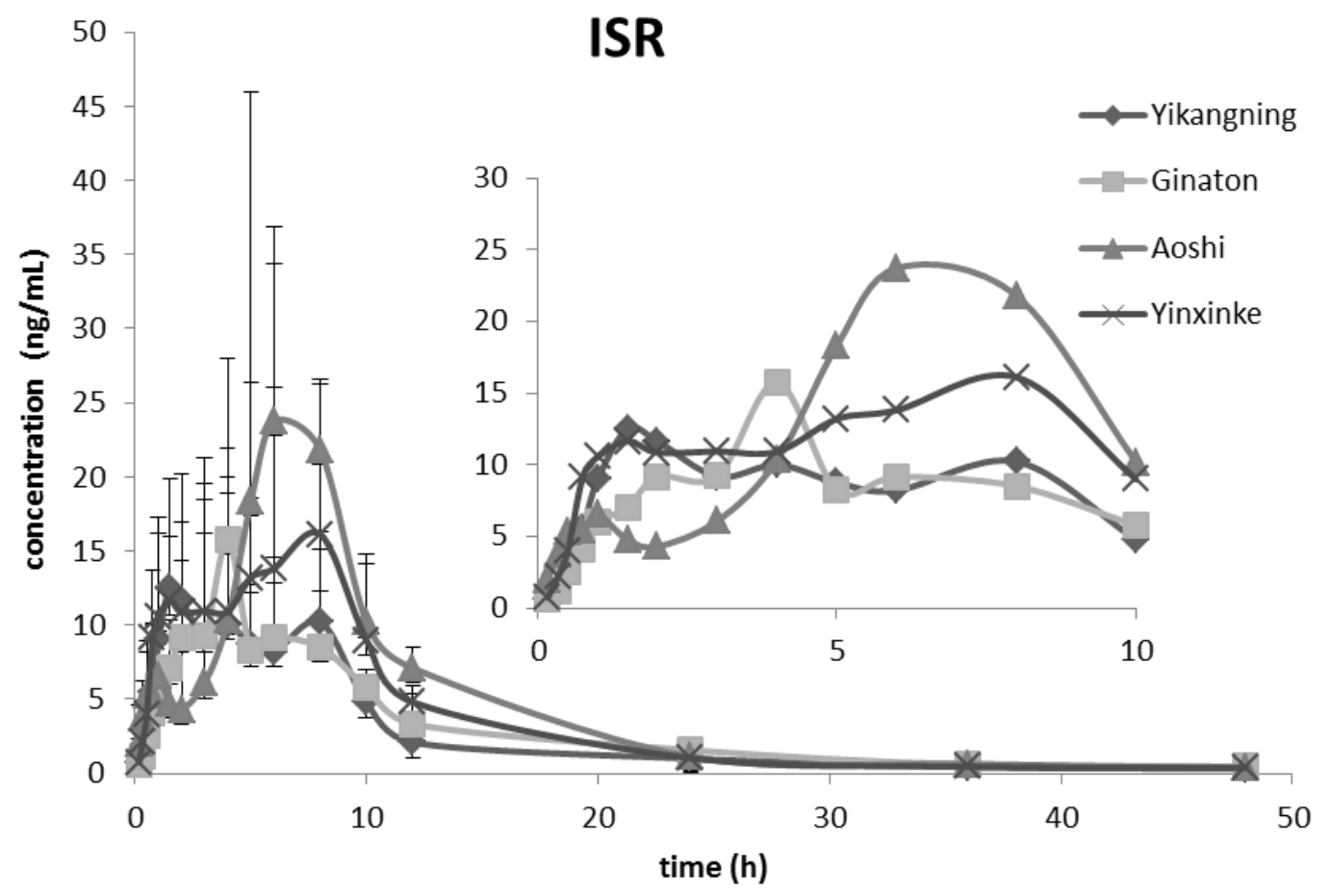

Fig 3: Concentration-time curve of isorhamnetin (ISR) for different preparations 


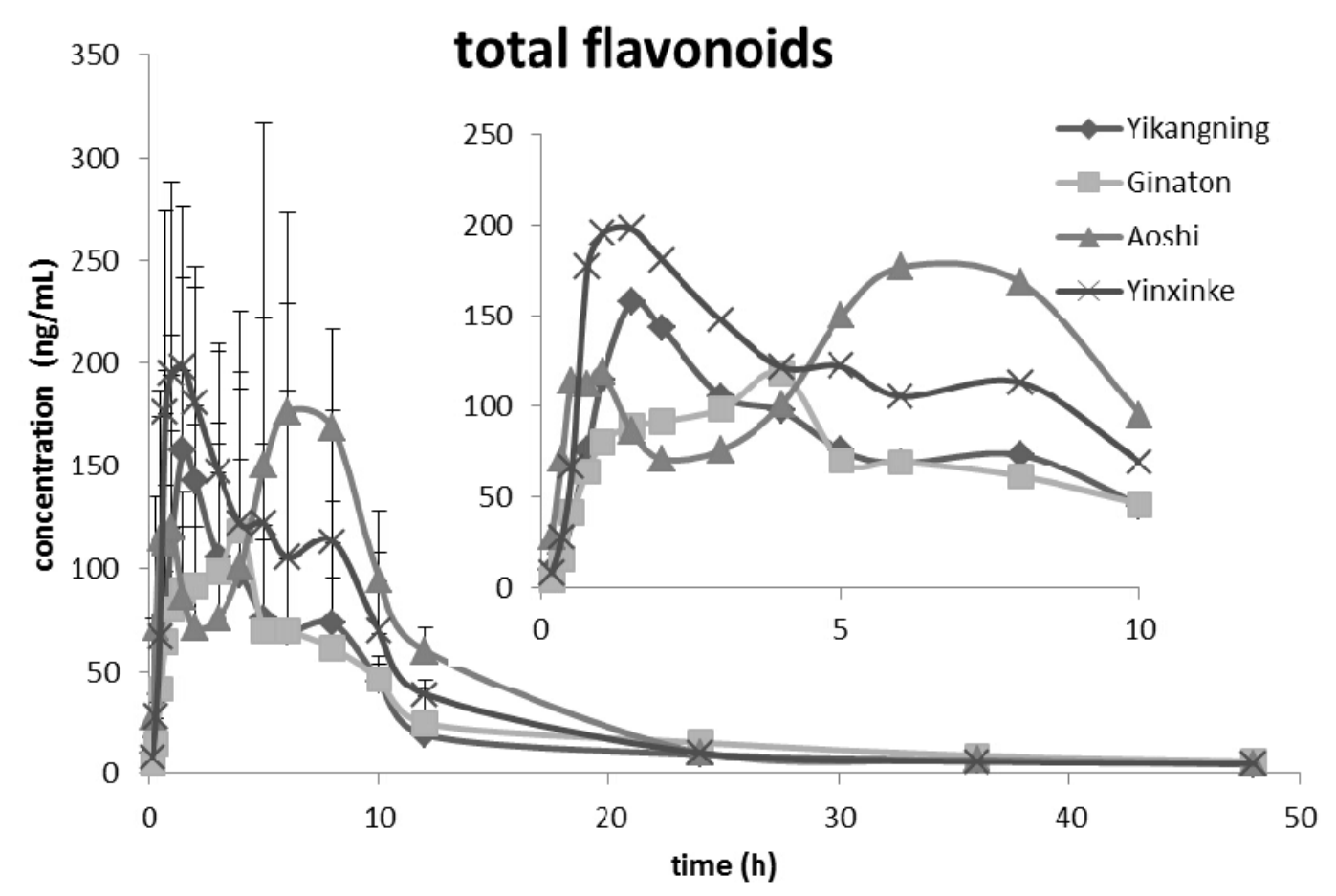

Fig 4: Concentration-time curve of total flavoniods for different preparations

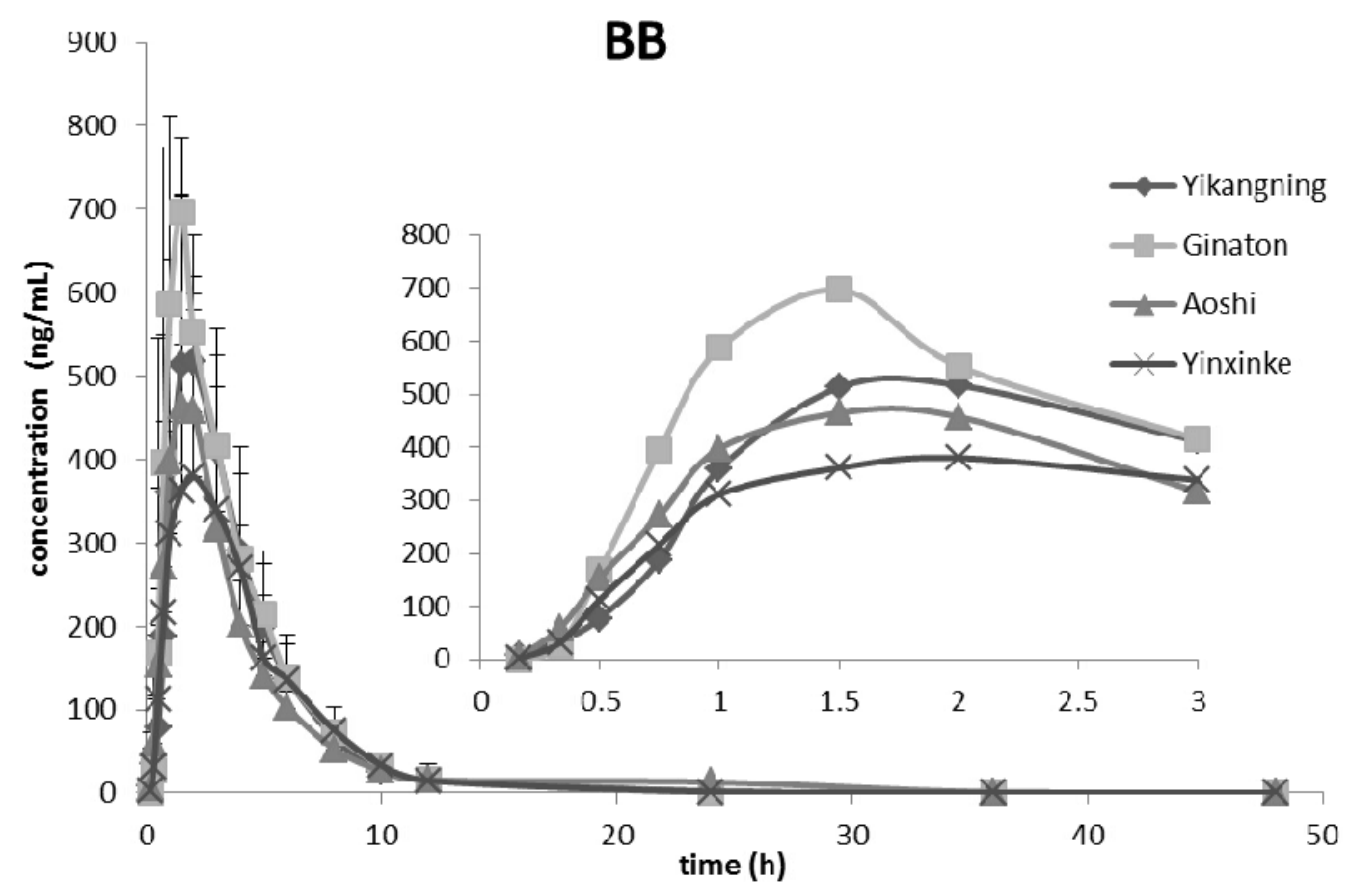

Fig 5: Concentration-time curve of bilobalide (BB) for different preparations 


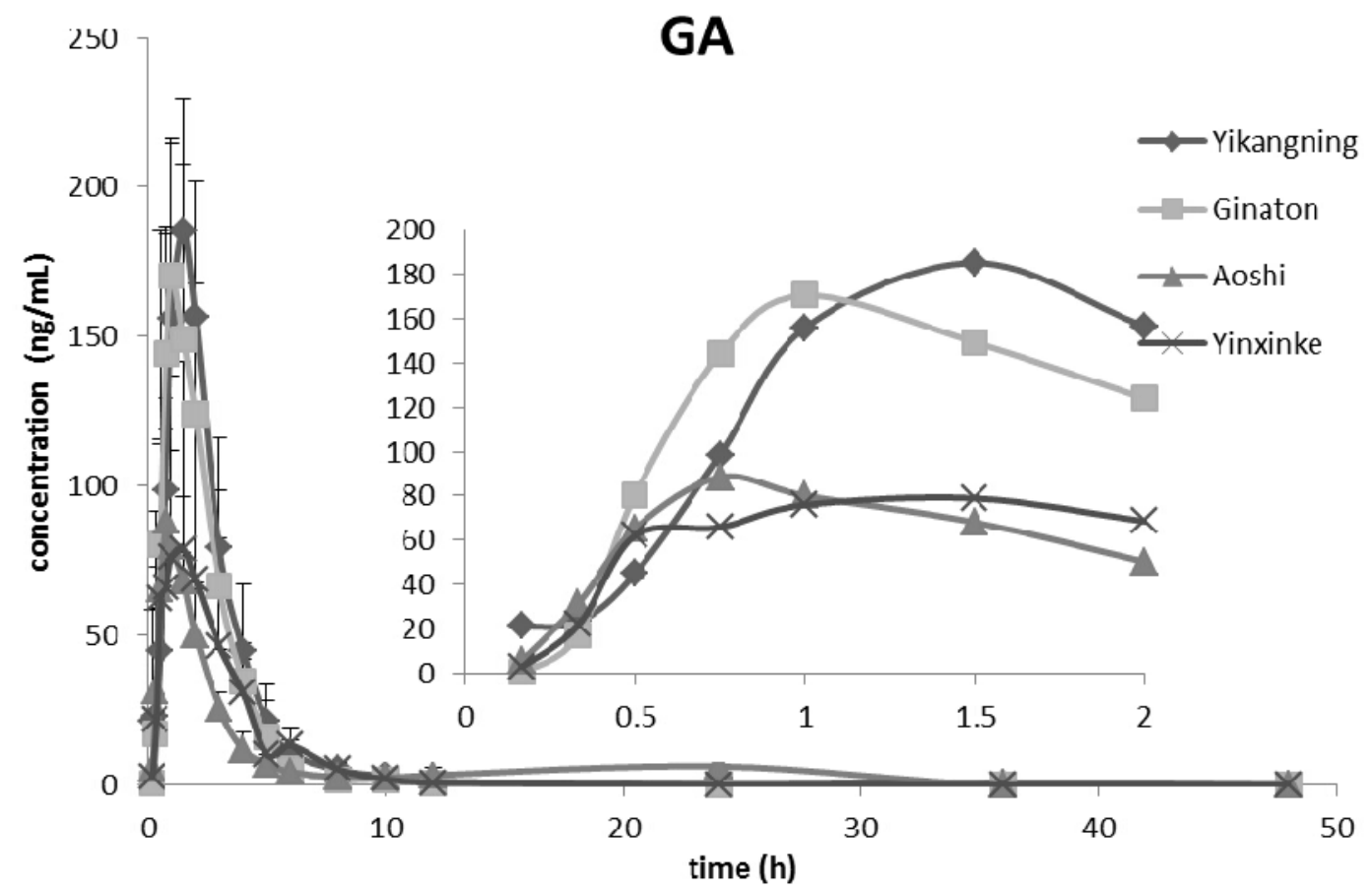

Fig 6: Concentration-time curve of ginkgolides A (GA) for different preparations

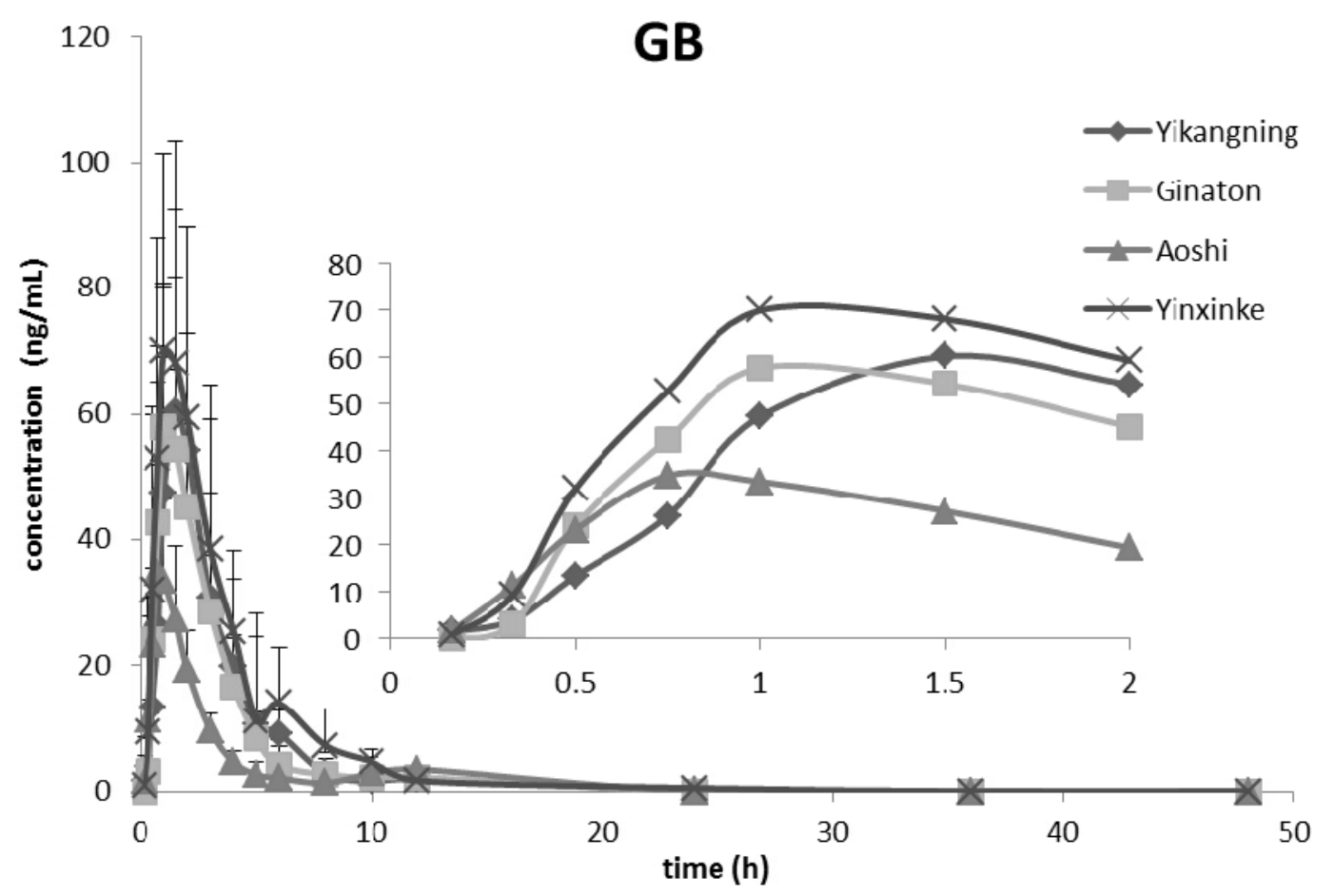

Fig 7: Concentration-time curve of ginkgolides B (GB) for different preparations 


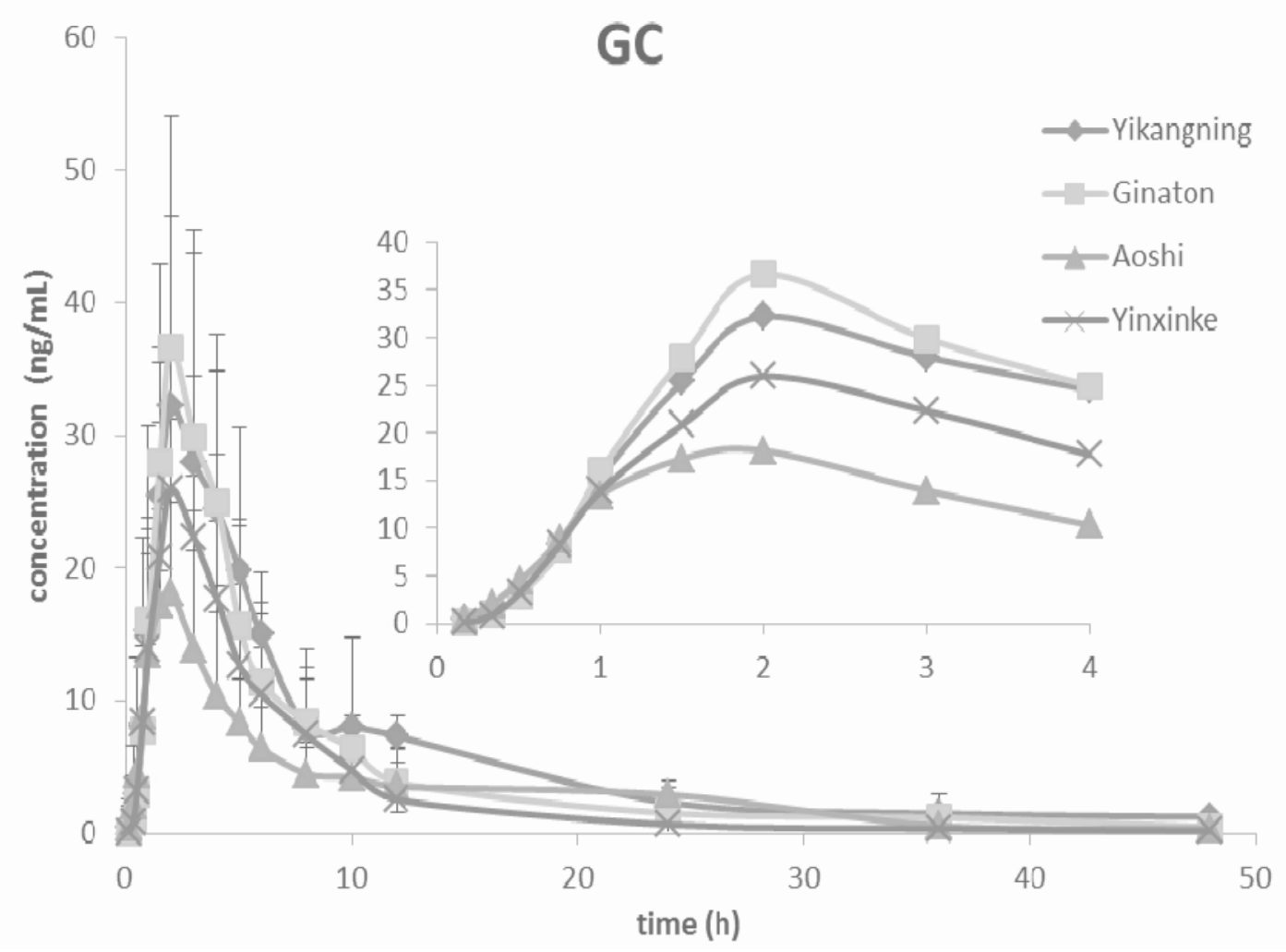

Fig 8: Concentration-time curve of ginkgolides $C(G C)$ for different preparations

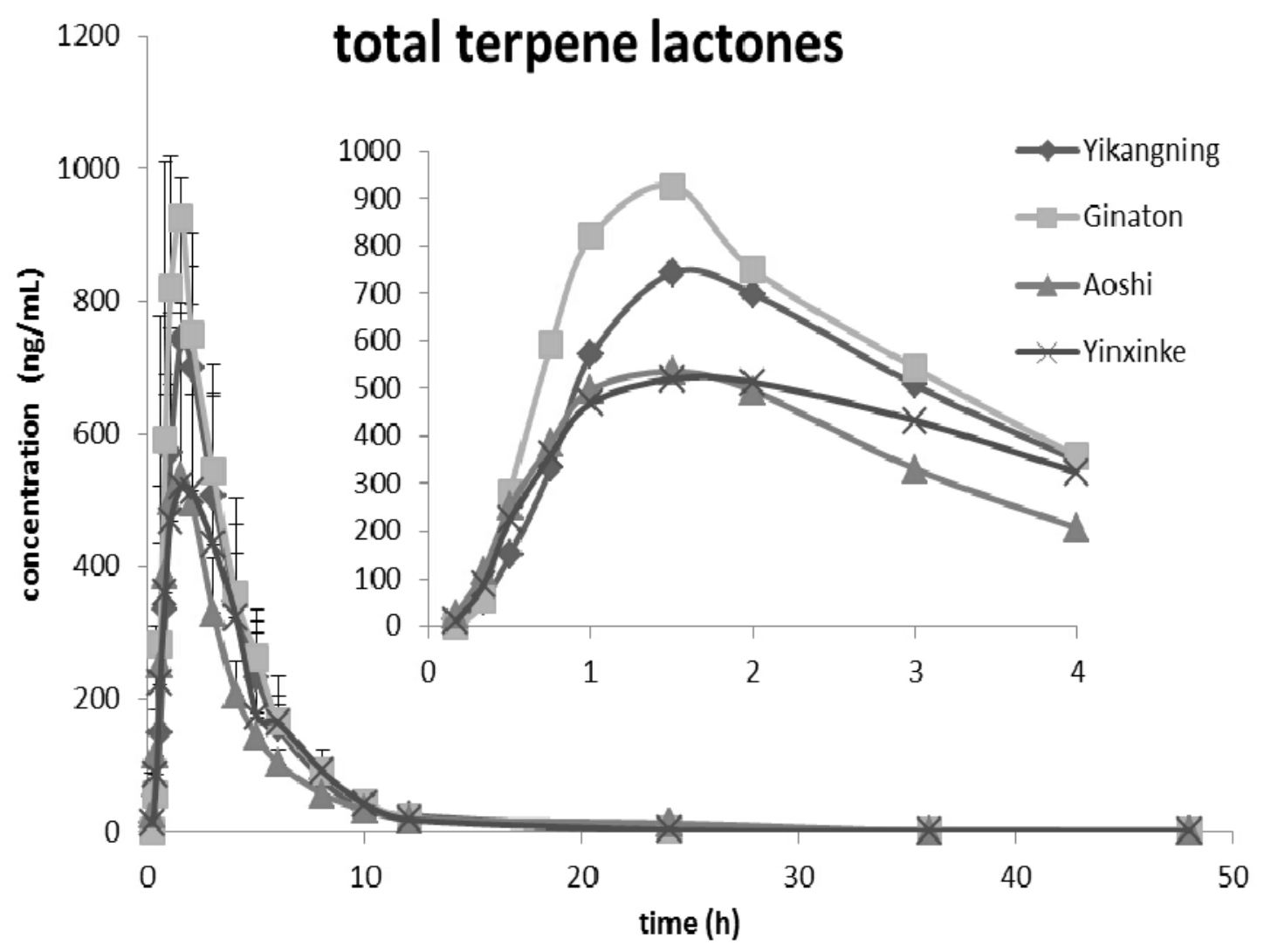

Fig 9: Concentration-time curve of total terpene lactones for different preparations 
Table 3: Major pharmacokinetic parameters (non-compartmental model) and relative bioavailability of flavonoids (KMF, QCT, ISR, and total flavonoids) after oral administration in the four GLP

\begin{tabular}{|c|c|c|c|c|c|c|}
\hline Flavonoid & Preparation & $\begin{array}{l}t_{1 / 2} \\
(h)\end{array}$ & $\begin{array}{l}t_{\max } \\
\text { (h) }\end{array}$ & $\begin{array}{c}C_{\max } \\
(\mu \mathrm{g} / \mathrm{L})\end{array}$ & $\begin{array}{c}\mathrm{AUC}_{0-\mathrm{t}} \\
(\mu \mathrm{g} / \mathrm{L} \cdot \mathrm{h})\end{array}$ & $\begin{array}{l}F_{r} \\
(\%)\end{array}$ \\
\hline \multirow{4}{*}{ KMF } & Yikangning & $21.76 \pm 7.94$ & $1.63 \pm 0.64$ & $26.97 \pm 17.15$ & $215.38 \pm 126.37$ & 97.15 \\
\hline & Ginaton & $17.02 \pm 7.91$ & $1.63 \pm 1.12$ & $14.42 \pm 4.96$ & $221.70 \pm 80.48$ & 100.00 \\
\hline & Aoshi & $11.68 \pm 4.57$ & $0.72 \pm 0.21$ & $18.15 \pm 12.68$ & $267.84 \pm 88.99$ & 120.81 \\
\hline & Yinxinke & $13.28 \pm 7.74$ & $1.34 \pm 0.48$ & $23.52 \pm 9.10$ & $221.25 \pm 95.03$ & 99.80 \\
\hline \multirow{4}{*}{ QCT } & Yikangning & $14.60 \pm 7.99$ & $1.53 \pm 0.71$ & $22.97 \pm 14.50$ & $151.60 \pm 124.33$ & 93.13 \\
\hline & Ginaton & $9.82 \pm 3.61$ & $1.83 \pm 1.43$ & $14.22 \pm 6.73$ & $162.78 \pm 111.66$ & 100.00 \\
\hline & Aoshi & $5.24 \pm 2.31$ & $0.75 \pm 0.35$ & $22.49 \pm 14.17$ & $275.85 \pm 158.87$ & 169.46 \\
\hline & Yinxinke & $12.07 \pm 6.75$ & $1.34 \pm 0.40$ & $45.31 \pm 25.47$ & $305.46 \pm 178.81$ & 187.65 \\
\hline \multirow{4}{*}{ ISR } & Yikangning & $10.60 \pm 3.91$ & $2.06 \pm 1.35$ & $12.54 \pm 8.31$ & $124.37 \pm 90.46$ & 87.41 \\
\hline & Ginaton & $10.97 \pm 5.07$ & $2.04 \pm 1.25$ & $15.75 \pm 12.06$ & $142.28 \pm 62.37$ & 100.00 \\
\hline & Aoshi & $6.13 \pm 3.33$ & $0.75 \pm 0.19$ & $6.53 \pm 4.66$ & $218.57 \pm 119.21$ & 153.62 \\
\hline & Yinxinke & $8.67 \pm 3.24$ & $2.22 \pm 1.76$ & $11.66 \pm 5.64$ & $182.00 \pm 160.10$ & 127.92 \\
\hline \multirow{4}{*}{$\begin{array}{l}\text { Total } \\
\text { flavonoids }\end{array}$} & Yikangning & $13.20 \pm 5.69$ & $1.50 \pm 0.76$ & $157.54 \pm 98.62$ & $1235.01 \pm 864.17$ & 96.53 \\
\hline & Ginaton & $10.29 \pm 2.90$ & $1.17 \pm 1.12$ & $118.31 \pm 72.48$ & $1279.36 \pm 644.11$ & 100.00 \\
\hline & Aoshi & $8.58 \pm 2.96$ & $0.69 \pm 0.22$ & $114.18 \pm 72.48$ & $1987.13 \pm 937.30$ & 155.32 \\
\hline & Yinxinke & $16.73 \pm 9.83$ & $1.34 \pm 0.40$ & $197.87 \pm 92.27$ & $1785.00 \pm 1063.65$ & 139.52 \\
\hline
\end{tabular}

Table 4: The main pharmacokinetic parameters (no compartmental) and the relative bioavailability of lactones (BB, GA, GB, GC, and total terpene lactones) after oral administration in the four GLP

\begin{tabular}{|c|c|c|c|c|c|c|}
\hline Lactone & Preparation & $\begin{array}{l}t_{1 / 2} \\
(h)\end{array}$ & $\begin{array}{c}T_{\max } \\
\text { (h) }\end{array}$ & $\begin{array}{c}C_{\max } \\
(\mu \mathrm{g} / \mathrm{L})\end{array}$ & $\begin{array}{c}\mathrm{AUC}_{0-\mathrm{t}} \\
(\mu \mathrm{g} / \mathrm{L} \cdot \mathrm{h})\end{array}$ & $\begin{array}{c}F_{r} \\
(\%)\end{array}$ \\
\hline & Yikangning & $3.43 \pm 1.54$ & $1.88 \pm 0.52$ & $517.15 \pm 202.96$ & $2261.87 \pm 812.35$ & 89.42 \\
\hline \multirow{3}{*}{$\mathrm{BB}$} & Ginaton & $2.02 \pm 0.51$ & $1.67 \pm 0.75$ & $697.56 \pm 224.22$ & $2529.46 \pm 320.48$ & 100.00 \\
\hline & Aoshi & $2.35 \pm 0.76$ & $1.44 \pm 0.42$ & $464.99 \pm 241.46$ & $1987.40 \pm 766.21$ & 78.57 \\
\hline & Yinxinke & $3.54 \pm 2.04$ & $1.75 \pm 0.60$ & $380.23 \pm 176.66$ & $2016.92 \pm 1150.92$ & 79.74 \\
\hline \multirow{5}{*}{ GA } & Yikangning & $1.60 \pm 1.07$ & $1.50 \pm 0.66$ & $185.19 \pm 60.37$ & $486.53 \pm 120.21$ & 116.08 \\
\hline & Ginaton & $1.07 \pm 0.45$ & $1.08 \pm 0.47$ & $170.44 \pm 40.07$ & $419.12 \pm 96.72$ & 100.00 \\
\hline & Aoshi & $2.19 \pm 1.75$ & $1.00 \pm 0.33$ & $88.52 \pm 48.80$ & $198.65 \pm 80.78$ & 47.40 \\
\hline & Yinxinke & $1.68 \pm 1.26$ & $1.03 \pm 0.51$ & $78.96 \pm 61.15$ & $271.68 \pm 248.38$ & 64.82 \\
\hline & Yikangning & $1.51 \pm 1.03$ & $1.41 \pm 0.71$ & $60.31 \pm 33.31$ & $186.37 \pm 154.12$ & 111.33 \\
\hline \multirow{3}{*}{ GB } & Ginaton & $2.14 \pm 1.24$ & $1.29 \pm 0.46$ & $57.80 \pm 28.23$ & $167.40 \pm 96.39$ & 100.00 \\
\hline & Aoshi & $2.41 \pm 1.21$ & $1.06 \pm 0.29$ & $34.85 \pm 19.27$ & $76.61 \pm 33.73$ & 45.76 \\
\hline & Yinxinke & $2.21 \pm 1.47$ & $1.22 \pm 0.41$ & $69.98 \pm 35.10$ & $245.23 \pm 160.49$ & 146.49 \\
\hline \multirow{4}{*}{ GC } & Yikangning & $7.97 \pm 4.04$ & $2.13 \pm 0.35$ & $32.21 \pm 11.19$ & $291.73 \pm 184.40$ & 137.56 \\
\hline & Ginaton & $5.50 \pm 2.82$ & $2.17 \pm 0.68$ & $36.65 \pm 15.03$ & $212.07 \pm 124.85$ & 100.00 \\
\hline & Aoshi & $5.55 \pm 2.19$ & $1.81 \pm 0.65$ & $18.14 \pm 8.33$ & $158.52 \pm 55.48$ & 74.75 \\
\hline & Yinxinke & $5.08 \pm 2.82$ & $2.06 \pm 0.42$ & $25.94 \pm 10.15$ & $160.49 \pm 104.66$ & 75.68 \\
\hline \multirow{4}{*}{$\begin{array}{l}\text { Total } \\
\text { terpene } \\
\text { lactones }\end{array}$} & Yikangning & $7.51 \pm 4.66$ & $1.63 \pm 0.58$ & $743.4 \pm 212.79$ & $3007.64 \pm 901.24$ & 88.36 \\
\hline & Ginaton & $3.47 \pm 2.47$ & $1.42 \pm 0.38$ & $926.71 \pm 198.67$ & $3403.77 \pm 295.20$ & 100.00 \\
\hline & Aoshi & $3.45 \pm 1.28$ & $1.34 \pm 0.48$ & $535.87 \pm 260.73$ & $2293.22 \pm 767.82$ & 67.37 \\
\hline & Yinxinke & $3.82 \pm 1.95$ & $1.50 \pm 0.76$ & $520.46 \pm 205.27$ & $2572.24 \pm 1453.58$ & 75.57 \\
\hline
\end{tabular}

To analyze $\mathrm{AUC}_{0-\mathrm{t}}, \mathrm{C}_{\max }$, and $\mathrm{T}_{\max }$ of flavonoids in the four different GLP with the LSD pairwise comparison methods, which reveal that, for KMF, there was no significant difference for $\mathrm{AUC}_{0-\mathrm{t}}$ of the four different GLP. For QCT, AUC $\mathrm{C}_{-\mathrm{t}}$ and $\mathrm{C}_{\max }$ of Yinxinke had a significant difference compared with Yikangning or Ginaton $(p<0.05)$; for ISR and total flavonoids, there were no significant differences for $A \cup C_{0-t}$ and $\mathrm{C}_{\max }$ of the four different GLP. The conclusion suggests that dispersible tablets can increase QCT absorption and bioavailability of QCT in vivo compared with traditional tablets. Besides, the Tmax of dropping pill Aoshi was found to be significantly lower among the four preparations.
To analyze $\mathrm{AUC}_{0-t}, \mathrm{C}_{\max }$, and $\mathrm{T}_{\max }$ of lactones in the four different GLP with the LSD pairwise comparison methods, which indicate that, for BB, there were no significant differences for $A \cup C_{0-t}$ of the four different GLP, but Ginaton had a higher $\mathrm{C}_{\max }$ compared with solid dispersions (Aoshi) and dispersible tablet (Yinxinke, $p<0.05$ ). For GA, there were higher $A U_{0-t}$ and $C_{\max }$ when Yikangning and Ginaton were compared with Aoshi and Yinxinke $(p<0.05)$. For GB, Aoshi had lower $\mathrm{AUC}_{0-\mathrm{t}}$ and $\mathrm{C}_{\max }$, when compared with Yikangning and Yinxingke $(p<0.05)$, the $\mathrm{C}_{\max }$ of Aoshi was also lower than them. For GC, there were higher $A \cup C_{0-t}$ and $\mathrm{C}_{\max }$ when Yikangning 
was compared with Aoshi $(p<0.05)$; the $\mathrm{C}_{\max }$ of Ginaton was also higher than Aoshi and Yinxingke $(p<0.05)$. For total lactones, Ginaton had higher $\mathrm{AUC}_{0-\mathrm{t}}$ and $\mathrm{C}_{\max }$, when compared with Aoshi $(p<0.05)$.

\section{DISCUSSION}

In this study, a four-period crossover trial was used in pharmacokinetic studies. The crossover design eliminates variability caused by subject (dog) differences in drug absorption, drug clearance, and the volume of drug distribution.

The plasma concentration-time curves of QCT, KMF, and ISR and total flavonoids in beagle dog plasma after administration of the four different GLP show double peaks. The findings are consistent with previously reports. Some researchers $[15,16]$ considered that it might be caused by some factors, such as enterohepatic circulation [7,17], distribution of the drug in vivo, and so on. To clarity the reason of double peaks of QCT, KMF, and ISR, a study on the absorption mechanism was performed [15]. The results demonstrated that the first peaks were produced by absorption-conjugation of the GBE-containing aglycones in the small intestine, and the second peaks were produced by the colonic deglycosylation-absorption-conjugation of the unabsorbed flavonoid glycosides.

In this study, the measured plasma concentrations of QCT, KMF, ISR, BB, GA, GB, and $\mathrm{GC}$ were multiplied by a conversion factor to calculate the corresponding plasma concentrations of each compound under the same dose. The results suggest that dispersible tablets and dropping pills can increase absorption and bioavailability of flavonoids (especially for QCT) in vivo compared with conventional tablet, and this has adverse consequences for lactones. Studies show that the lactones are unstable under acidic conditions, and therefore, preparations with good disintegrating property may cause greater degradation of the drugs [18].

\section{CONCLUSION}

Among these preparations, Yinxinke, a dispersible tablet improved the $\mathrm{AUC}_{0-\mathrm{t}}$ and $\mathrm{C}_{\max }$ of quercetin, while Aoshi, the dropping pill shortened the $T_{\max }$ of all the flavonoids. Good disintegrating property is helpful to the absorption of flavonoids, but may be disadvantageous for lactones compared with conventional preparations.

\section{ACKNOWLEDGEMENT}

This work was supported by the National Natural Science Foundation of China (81073057), Innovation Team Development Program of Beijing University of Chinese Medicine (nos. 2011-CXTD-13) and National Basic Research Program of China (973 Program, no. $2012 \mathrm{CB} 724000$.

\section{REFERENCES}

1. Satoh $H$ and Nishida S. Electropharmacological actions of Ginkgo biloba extract on vascular smooth and heart muscles. Clin. Chim. Acta, 2004; 1-2: 13-22.

2. Kennedy DO, Haskell CF, Mauri PL and Scholey AB. Acute cognitive effects of standardised Ginkgo biloba extract complexed with phosphatidylserine. Hum. Psycho pharm. Clin. 2007; 4: 199-210.

3. Koh PO. Identification of proteins differentially expressed in cerebral cortexes of Ginkgo biloba extract (EGb761)-treated rats in a middle cerebral artery occlusion model--a proteomics approach. The American Journal of Chinese Medicine. 2011; 2: 315324.

4. Lang $D$, Ude $C$, Wurglics $M$, Schubert-Zsilavecz $M$ and Klein J. Brain permeability of bilobalide as probed by microdialysis before and after middle cerebral artery occlusion in mice. J. Pharm. Pharm. Sci. 2010; 4: 607-614.

5. Ude C, Schubert-Zsilavecz $M$ and Wurglics M. Ginkgo biloba Extracts: A Review of the Pharmacokinetics of the Active Ingredients. Clincal Pharmacokineties. 2013; 9: 727-749.

6. Westman J, Drieu $K$ and Sharma HS. Antioxidant compounds EGB-761 and BN-520 21 attenuate heat shock protein (HSP $72 \mathrm{kD}$ ) response, edema and cell changes following hyperthermic brain injury. An experimental study using immunohistochemistry in the rat. Amino Acids. 2000; 1: 339-350.

7. Drago F, Floriddia ML, Cro $M$, Giuffrida $S$. Pharmacokinetics and bioavailability of a Ginkgo biloba extract. J Ocul Pharmacol Th. 2002; 2: 197202.

8. Li L, Zhao Y, Du F, Yang J, Xu F, Niu W, Ren Y and Li C. Intestinal absorption and presystemic elimination of various chemical constituents present in GBE50 extract, a standardized extract of Ginkgo biloba leaves. Current Drug Metabolism. 2012; 5: 494-509.

9. Woelkart K, Feizlmayr E, Dittrich P, Beubler E, Pinl F, Suter A, Bauer R. Pharmacokinetics of bilobalide, ginkgolide $A$ and $B$ after administration of three different Ginkgo biloba L. preparations in humans. Phytother Res. 2010; 3: 445-450.

10. Chen ZP, Sun J, Chen HX, Xiao YY, Liu D, Chen J, Cai $H$, Cai BC. Comparative pharmacokinetics and bioavailability studies of quercetin, kaempferol and isorhamnetin after oral administration of Ginkgo 
biloba extracts, Ginkgo biloba extract phospholipid complexes and Ginkgo biloba extract solid dispersions in rats. Fitoterapia. 2010; 8: 1045-1052.

11. Chen F, Li L, Xu F, Sun Y, Du F, Ma X, Zhong C, Li X, Wang $F$, Zhang $N, L i C$. Systemic and cerebral exposure to and pharmacokinetics of flavonols and terpene lactones after dosing standardized Ginkgo biloba leaf extracts to rats via different routes of administration. Brit J Pharmacol. 2013a; 2: 440-457.

12. Rangel-Ordonez $L$, Noldner $M$, Schubert-Zsilavecz $M$, Wurglics M. Plasma levels and distribution of flavonoids in rat brain after single and repeated doses of standardized Ginkgo biloba extract EGb 761(R). Planta Med. 2010; 15: 1683-1690.

13. Rossi R, Basilico F, Rossoni G, Riva A, Morazzoni $P$, Mauri PL. Liquid chromatography/atmospheric pressure chemical ionization ion trap mass spectrometry of bilobalide in plasma and brain of rats after oral administration of its phospholipidic complex. $J$ Pharmaceut and Biomed. 2009; 2: 224-227.

14. Zhang $Q$, Wang GJ, A JY, Wu D, Zhu LL, Ma B, Du Y. Application of GC/MS-based metabonomic profiling in studying the lipid-regulating effects of Ginkgo biloba extract on diet-induced hyperlipidemia in rats. Acta Pharmacol Sin. 2009; 12: 1674-1687.

15. Chen F, Li L, Xu F, Sun Y, Du F, Ma X, Zhong C, Li X, Wang $F$, Zhang N, Li C. Systemic and cerebral exposure to and pharmacokinetics of flavonols and terpene lactones after dosing standardized Ginkgo biloba leaf extracts to rats via different routes of administration. Brit J Pharmacol. 2013b; 2: 440-457.

16. Zhao $Y$, Sun $Y, \mathrm{Li}$ C. Simultaneous determination of ginkgo flavonoids and terpenoids in plasma: ammonium formate in LC mobile phase enhancing electrospray ionization efficiency and capacity. J Am SOC Mass Spectrom. 2008; 3: 445-449.

17. Liu Y, Liu Y, Dai Y, Xun L, Hu M. Enteric disposition and recycling of flavonoids and ginkgo flavonoids. J Altern Complem Med. 2003; 5: 631-640.

18. Lin CY, Liang LJ, Zeng CM. Study on Stability of Terpene Lactones from Ginkgo Leaf. Tradit Chin Drug Res Clin Pharmacol. 2013, 24: 622-624. 